\title{
Studies of light backscattering at concrete during its hydration
}

\author{
Gorsky M.P., Maksimyak P.P. and Maksimyak A.P. \\ Correlation Optics Department, Chernivtsi National University \\ 2 Kotsyubynsky St., Chernivtsi, Ukraine; E-mail: misha@itf.cv.ua
}

Received: 30.03.2009

\begin{abstract}
In this work we have studied, both theoretically and experimentally, changes in the integral reflection coefficient of concrete during its hydration. A diffuse approximation method has been used for description of light scattering at concrete during its hydration and the results have been compared with the experimental data obtained. The optical diagnostics technqiue introduced by us allows performing diagnostics of earlier hydration stages in the concrete hardening process and predicting mechanical properties of the concrete produced.
\end{abstract}

Keywords: backscattering, diffuse approximation, concrete hydration.

PACS: 41.85.Ja; 42.25.Bs

UDC: 535.36

\section{Introduction}

Concretes are basic materials of modern construction industry and basic backfill materials for oil-and-gas industry. That is why it is important to predict beforehand their mechanical properties and solidification rate of the concretes composed. Formation of concrete passes through a process of hydration, interaction of cement and water. According to existing models, concrete induration occurs due to enlargement and accretion of powder cement particles [1-5]. The beginning and the end of induration of concrete solution is usually determined [6,7] using Vicat apparatus. It measures immersion depth of needle in normal cement solution poured into a special taper. According to the Vicat technique, induration begins when needle does not reach taper bottom for $10 \mathrm{~mm}$, and finishes when needle dips into the solution for less than $3 \mathrm{~mm}$. However, the technique does not allow judging impartially hydration stages and cement induration, as well as predicting mechanical properties of the concrete produced.

Optical methods for diagnostics of concrete hydration have a great potential $[8,9]$. In this work we investigate theoretically and experimentally changes in the integral reflection coefficient (IRC) of concrete during its hydration. We reveal that the IRC changes are related to concrete hydration and that the character of these changes allows predicting mechanical properties of the concrete produced.

At the beginning of hydration process, cement solution consists of gel, free water and unhydrated cement particles. Then formation of crystals is generated, which join the system and lead to increasing cement solution viscosity. The hydration process becomes 
completed with full induration of the cement solution [1-4]. The time of hydration depends on specific cement powder mix, cement particle sizes and water amount.

Cement is a complicated mixture of particles with different sizes and shapes, which consist by $95-97 \%$ of oxides $\mathrm{Ca}, \mathrm{Si}_{2}, \mathrm{Al}_{2} \mathrm{O}_{3}$ and $\mathrm{Fe}_{2} \mathrm{O}_{3}$. These compounds constitute some minerals, of which the main are as follows [1-4]:

- $\quad$ tricalcic silicate (alite) $3 \mathrm{CaO} \cdot \mathrm{SiO}_{2}(40-65 \%)$;

- $\quad$ dicalcic silicate (belite) $2 \mathrm{CaO} \cdot \mathrm{SiO}_{2}(15-45 \%$ );

- tricalcic aluminate $3 \mathrm{CaO} \cdot \mathrm{Al}_{2} \mathrm{O}_{3}(4-12 \%)$;

- tetracalcic alumoferrite $4 \mathrm{CaO} \cdot \mathrm{Al}_{2} \mathrm{O}_{3} \cdot \mathrm{Fe}_{2} \mathrm{O}_{3}(12-25 \%)$;

- $\quad$ gypsum.

Cement particle size distribution may differ not only for various kinds of cement, but for different consignments, too. Measurements of the particle size distribution are performed with different methods, such as Electrical Zone Sensing, Sedimentation and Scanning Electron Microscopy [1-5]. A standard procedure is to measure remains weight on a sieve while screening consecutively from the biggest mesh aperture to the smallest. Laser diffraction method [5] is used for studying real-time distribution of cement particle sizes.

Backscattering is a promising direction in investigations of concrete properties and its hydration. Photos of concrete cuts $[1,2]$ show that the concrete should be considered as a disperse medium (DM). For mathematical description of light scattering at DMs, analytical methods and transport theory have been used [10-14]. In particular, small angle approximation allows getting an analytical solution for a radar probe of DM and radiation scattering at mobile particles [10]. Analytical solutions have been derived for the backscattering by the media consisting of equal-size spheres [10, 12]. For multiple scattering in DM, the transport theory provides quite good results, in particularly a socalled diffuse approximation method $[10,13,14]$. We have used this method in order to describe the light scattering in concrete during its hydration and have compared its results with the experimental data obtained by us.

\section{Light scattering in disperse medium in frame of the diffuse approximation}

Let us analyse light scattering in a disperse medium in frame of the diffuse approximation following considerations developed in the works $[10,14]$. For a medium with the bulk concentration (i.e., the ratio of the volume of particles and the entire system volume) exceeding $1 \%$, the method of diffuse approximation provides the best fit. In this case the intensity of radiation in randomly heterogeneous medium consists of two parts: a regular constituent of output radiation $I_{r i}$ and a diffuse scattered radiation $I_{d}$. If any internal sources are absent at a point $\vec{r}$, then $I_{d}$ should satisfy the transport equation:

$$
\begin{aligned}
& \frac{d I_{d}(\vec{r}, \vec{s})}{d v} \\
& =-\rho_{c} \sigma_{t 0} I_{d}(\vec{r}, \vec{s})+\frac{\rho_{c} \sigma_{t 0}}{4 \pi}\left[\int_{4 \pi} \rho_{f}\left(\vec{s}, \vec{s}^{\prime}\right) I_{d}\left(\vec{r}, \vec{s}^{\prime}\right) d \omega^{\prime}+\int_{4 \pi} \rho_{f}\left(\vec{s}, \vec{s}^{\prime}\right) I_{r i}\left(\vec{r}, \vec{s}^{\prime}\right) d \omega^{\prime}\right],
\end{aligned}
$$


where $d v$ is the volume element surrounding the point $\vec{r}, \sigma_{t 0}$ the particle extinction section, $\rho_{c}$ the absolute concentration of particles, $\frac{1}{4 \pi} \rho_{f}\left(\vec{s}, \vec{s}^{\prime}\right)$ denotes a dimensionless quantity, a so-called "phase function" (it describes the radiation intensity scattered at a particle in the $\vec{s}^{\prime}$ direction, while a plane wave falls upon it in the $\vec{s}$ direction), $I_{d}(\vec{r}, \vec{s})$ the energy flux density of diffusely scattered radiation at the point $\vec{r}$ in the $\vec{s}$ direction, $I_{r i}(\vec{r}, \vec{s})$ the energy flux density of regular radiation component at the point $\vec{r}$ in the $\vec{s}$ direction, and $d \omega^{\prime}$ the solid angle element in the $\vec{s}^{\prime}$ direction.

In any complicated medium, the diffuse scattering seems to be isotropic due to multiple reflections at many particles, i.e. $I_{d}(\vec{r}, \vec{s})=U_{d}(\vec{r})$, where

$$
U_{d}(\vec{r})=\frac{1}{4 \pi} \int_{4 \pi} I_{d}(\vec{r}, \vec{s}) d \omega
$$

is the average intensity of the diffusely scattered radiation (see Fig. 1) and $d \omega$ the solid angle element in the $\vec{s}$ direction. For simplified consideration of radiation anisotropy one can introduce the parameter

$$
I_{d}(\vec{r}, \vec{s})=U_{d}(\vec{r})+c F_{d}(\vec{r}) \vec{s},
$$

where $c$ is a certain constant and the parameter,

$$
F_{d}(\vec{r})=\int_{4 \pi} I_{d}(\vec{r}, \vec{s}) \vec{s} d \omega=F(\vec{r}) \vec{s}_{f},
$$

represents a so-called diffuse flux vector, which shows deviation of the diffuse scattering from isotropic one occurring for the plane wave propagating in the $\vec{s}_{f}$ direction (see Fig. 1). Let us rewrite Eq. (3) as

$$
I_{d}(\vec{r}, \vec{s})=U_{d}(\vec{r})+c F(\vec{r}) \vec{s}_{f} \times \vec{s}
$$

and insert it in Eq. (4):

$$
F(\vec{r}) \vec{s}_{f} \vec{s}_{f}=\int_{4 \pi} U_{d}(\vec{r}) \vec{s} d \omega+c \int_{4 \pi} F(\vec{r})\left(\vec{s}_{f} \times \vec{s}\right)^{2} d \omega .
$$

The first integral in Eq. (6) is equal to zero according to definition of the $U_{d}(\vec{r})$ function for isotropic distribution. The second integral is quite easy to calculate by directing the polar axes of spherical coordinate system along $\vec{s}_{f}$ and considering that the variables $\vec{s}_{f}$ and $\vec{r}$ are independent. Taking into account that $\vec{s}_{f}$ is a unit vector, we could get

$$
F(\vec{r})=c F(\vec{r}) \int_{0}^{\pi} d \theta \int_{0}^{2 \pi} d \varphi \cos ^{2} \theta \sin \theta=\frac{4 \pi}{3} c F(\vec{r}) .
$$

Hence, the constant $c$ is equal to $c=3 / 4 \pi$. 


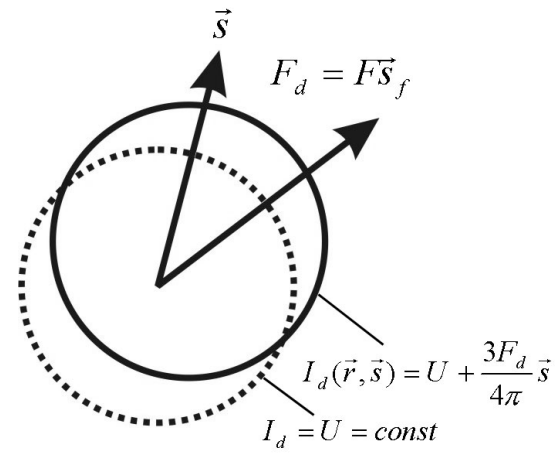

Fig. 1. Representation of scattered intensity in the diffuse approximation [10].

According to Fig. 1, then the scattered intensity within the diffuse approximation could be written as

$$
I_{d}(\vec{r}, \vec{s})=U_{d}(\vec{r})+\frac{3 F_{d}(\vec{r})}{4 \pi} \vec{s} .
$$

After substituting Eq. (8) in Eq. (1), the finite transport equation in our approximation would be as follows [10]:

$$
\begin{aligned}
& \nabla^{2} U_{d}(\vec{r})-\chi_{d}^{2} U_{d}(\vec{r}) \\
& =-3 \rho_{c} \sigma_{s} \rho_{c} \sigma_{t r} U_{r i}(\vec{r})+\frac{3}{4 \pi} \int_{4 \pi} \int_{4 \pi} \rho_{f}\left(\vec{s}, \vec{s}^{\prime}\right) I_{r i}\left(\vec{r}, \vec{s}^{\prime}\right) d \omega^{\prime} \vec{s} d \omega^{\prime}
\end{aligned}
$$

where $\chi_{d}^{2}=3\left(\rho_{c} \sigma_{a}\right)\left(\rho_{c} \sigma_{t r}\right), \sigma_{a}$ means the section of light absorption by the particle, $\sigma_{t r} \rightarrow \sigma_{s 0}(1-H)(1-\bar{\mu})+\sigma_{a}$ the transport section, $\sigma_{s}=\sigma_{s 0}(1-H)$ the corrected scattering section, $\sigma_{s 0}$ the section of light scattering by the particle, $H$ the ratio of the volume of particles and the entire system volume, $U_{r i}(\vec{r})=\frac{1}{4 \pi} \int_{4 \pi} I_{r i}(\vec{r}, \vec{s}) d \omega$, and $\bar{\mu}=\left(\int_{4 \pi} \rho_{f}\left(\vec{s}, \vec{s}^{\prime}\right) \vec{s} \times \vec{s}^{\prime} d \omega^{\prime}\right) /\left(\int_{4 \pi} \rho_{f}\left(\vec{s}, \vec{s}^{\prime}\right) d \omega^{\prime}\right)$ is the average cosine of the scattering angle.

The latter describes normalised difference between the average forward and backward scattering at a single particle and characterises quantitatively the scattering anisotropy. The parameter $p=(1-H)$ determines the porosity of the medium and is often used for its characterisation. Hereafter we will use just this parameter. In particular, the value $p=0$ means that the medium is absolutely solid, whereas $p=1$ characterises absolutely rarefied one.

As the scattering does not take place out of the medium, one of the boundary conditions of Eq. (9) is that the diffuse flux from the outside to inside medium should be equal to zero on its surface: 


$$
\int_{2 \pi} I_{d}(\vec{r}, \vec{s})(\vec{s} \times \vec{n}) d \omega=0,
$$

where $\vec{n}$ is the normal to the surface of medium at the point $\vec{r}$. Here the integration is carried out over the half-plane borders of the outside medium, i.e. over the half of full solid angle. This boundary condition, according [10], describes fairly well the scattering when the scattering section is equal or larger than the absorbing section. After integration, we could write Eq. (10) as

$$
U_{d}\left(\vec{r}_{s}\right)-\frac{2}{3 \rho_{c} \sigma_{t r}} \frac{\partial}{\partial n} U_{d}\left(\vec{r}_{s}\right)+\frac{2 \vec{n} Q\left(\vec{r}_{s}\right)}{4 \pi}=0,
$$

where $Q(\vec{r})=\frac{\sigma_{t}}{\sigma_{t r}} \int_{4 \pi}\left(\frac{1}{4 \pi} \int_{4 \pi} \rho_{f}\left(\vec{s}, \vec{s}^{\prime}\right) \vec{s} d \omega\right) I_{r i}\left(\vec{r}, \vec{s}^{\prime}\right) d \omega^{\prime} \quad$ describes the influence of anisotropy on the scattering process and $\vec{r}_{s}$ is the point on the medium surface.

When the plane wave is incident upon a layer of scatterers, diminution in the regular constituent occurs:

$$
I_{r i}=I_{0} \exp \left(-\rho_{c} \sigma_{t 0} L\right),
$$

where $L$ denotes the thickness of the layer of scatterers and $\sigma_{t 0}$ the extinction section of the particles. Then the following formulae are valid for the effective values of absorbing, scattering and extinction sections of the particle system [10]:

$$
\sigma_{a}=\int_{0}^{\infty} \sigma_{a}(a) W(a) d a, \sigma_{s 0}=\int_{0}^{\infty} \sigma_{s 0}(a) W(a) d a, \sigma_{t 0}=\int_{0}^{\infty} \sigma_{t 0}(a) W(a) d a,
$$

where $W(a)$ is the function of particle size distribution.

According to the method, the coefficient of diffuse reflection at the disperse semibounded medium does not depend on the absolute concentration of scatterers. It is determined by the medium porosity, scattering anisotropy, the transport, absorbing, scattering and the extinction sections and could be written as

$$
R_{d}=-\frac{3 \sigma_{s}\left[\bar{\mu} \sigma_{t} \sqrt{3 \sigma_{a} \sigma_{t r}}+\sigma_{t r} \sqrt{3 \sigma_{a} \sigma_{t r}}-3 \bar{\mu} \sigma_{a} \sigma_{t r}-\sigma_{t} \sigma_{t r}\right]}{\left(3 \sigma_{a} \sigma_{t r}-\sigma_{t}^{2}\right)\left(3 \sigma_{t r}+2 \sqrt{3 \sigma_{a} \sigma_{t r}}\right)} .
$$

In this formula, $\sigma_{s}, \sigma_{t r}$ and $\sigma_{t}$ represent the corrected scattering section, transport section and the corrected extinction section, respectively. Using the porosity, they could be defined in the following form:

$$
\begin{aligned}
& \sigma_{s}=\sigma_{s 0} p, \\
& \sigma_{t r}=\sigma_{s 0} p(1-\bar{\mu})+\sigma_{a}, \\
& \sigma_{t}=\sigma_{s 0} p+\sigma_{a} .
\end{aligned}
$$

Eq. (14) is original and has never been cited before in the literature.

Notice that for ensuring the semi-bounded medium approximation, the layer of scatterers has to diminish the regular constituent of radiation by 100 times at the least. 
From Eq. (14) it follows that $R_{d}=-1$ for nonabsorbing scatterers. This means that the nonabsorbing porous medium could transmit all the incident light due to multiple scattering. The minus sign corresponds to the opposite directions of the incident and scattered electromagnetic radiation fluxes. On the other hand, if scatterers are absorbing, we have $R_{d}=0$ for absolutely solid medium. According to the Fresnel formulae, the boundary between the media should reflect inversely, though Eq. (14) does not take it into consideration. Denoting the reflection from the surface as $R_{f}$, we could write the overall reflection coefficient as

$$
R=R_{d}+R_{f} .
$$

A considerable advantage of the diffuse approximation method is that it can involve the scattering anisotropy, which could be essential in case of multiple scattering. Nonetheless, the method does not take into account the effect of amplification for the coherent radiation scattered strictly backward [12]. It is also necessary to note that, in the case of concrete, it is very difficult to include the coherent amplification effects at all.

\section{Calculation of scattering and absorbing sections}

While using Eq. (14), we have to calculate the corresponding sections according to Eqs. (15)-(17). It is known that only spherical, cylindrical and spheroidal sections have been mathematically treated up to date. However, the cement particles may have various complicated shapes, so that it would be difficult to prefer a single special shape only.

We have calculated the extinction sections $\sigma_{t 0}=\sigma_{s 0}+\sigma_{a}$ and the scattering one $\sigma_{s 0}$ for the single scatterers using the Mie theory [10, 11], which describes scattering of electromagnetic waves at a single spherical scatterer on the basis of classical electrodynamics. According to this theory, the quantities mentioned above may be defined as

$$
\begin{aligned}
& \frac{\sigma_{t 0}}{\pi a^{2}}=2 x^{-2} \sum_{k=1}^{\infty}(2 k+1) \operatorname{Re}\left(a_{k}+b_{k}\right), \\
& \frac{\sigma_{s 0}}{\pi a^{2}}=2 x^{-2} \sum_{k=1}^{\infty}(2 k+1)\left(\left|a_{k}\right|^{2}+\left|b_{k}\right|^{2}\right) .
\end{aligned}
$$

Here $a$ is the radius of the scatterer, which is considered to be spherical for simplicity, and $x=2 \pi a / \lambda$, where $\lambda$ denotes the light wavelength. The terms in the infinite series involved in Eqs. (19) and (20) could be found as

$$
\begin{aligned}
& a_{k}=\frac{\psi_{k}(x) \psi_{k}^{\prime}(m x)-m \psi_{k}(m x) \psi_{k}^{\prime}(x)}{\zeta_{k}(x) \psi_{k}^{\prime}(m x)-m \psi_{k}(m x) \zeta_{k}^{\prime}(x)}, \\
& b_{k}=\frac{m \psi_{k}(x) \psi_{k}^{\prime}(m x)-\psi_{k}(m x) \psi_{k}^{\prime}(x)}{m \zeta_{k}(x) \psi_{k}^{\prime}(m x)-\psi_{k}(m x) \zeta_{k}^{\prime}(x)},
\end{aligned}
$$


where $m$ is the complex relative refractive index of scatterer's substance, strokes mean differentiation, and the functions $\psi_{k}(x)$ and $\zeta_{k}(x)$ are defined through the Bessel function of first kind $J_{k+1 / 2}(x)$ of real argument and the Hankel function of first kind $I_{k+1 / 2}^{(1)}(x)$ in the following way:

$$
\begin{aligned}
& \psi_{k}(x)=\sqrt{\frac{\pi x}{2}} J_{k+1 / 2}(x), \\
& \zeta_{k}(x)=\sqrt{\frac{\pi x}{2}} H_{k+1 / 2}^{(1)}(x) .
\end{aligned}
$$

In our model calculations all the scatterers have been regarded as spherical. The refractive index of cement materials is complex $(m=n+i \chi)$, with its real and imaginary parts diverging in different works as much as $n=1.5-1.7$ and $\chi=0.003-1$ [1-5]. Finally, the average particle radius has been taken as $2.4-14 \mu \mathrm{m}$.

Essential discord for the imaginary part of the refractive index found in different sources has led us to studies of the cement used in our experiments. With this aim, thin samples of cement powder suspended in immersion liquid with $n=1.51$, which remains neutral to cement, have been made. The thicknesses of samples have been within $40-50 \mu \mathrm{m}$. The samples have been measured using the setup, of which optical scheme is represented in Fig. 2. Using this scheme, we have measured the regular constituent of the scattered radiation intensity with the aid of light detector 3 . The integral diffuse reflection and scattering coefficients have been measured with an integral sphere 1 and a light detector 4 .

For the case of single scattering, the absorbed radiation is determined as

$$
I \approx I_{0} \exp \left(-\rho_{c} \sigma_{a} L\right)
$$

Therefore, when the scattering remains too small, decrease in the regular constituent occurs due to radiation absorption by the particles. It is clear that the radiation absorption during multiple scattering also occurs, due to absorbing properties of the particles. Moreover, the track of multiply scattered radiation would be always longer than the

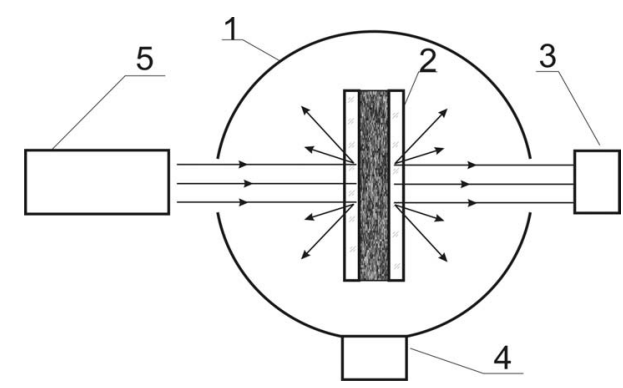

Fig. 2. Scheme for optical studies of thin cement samples: $1-$ integral sphere, 2 - sample, 3 and 4 - light detectors and 5 - He-Ne laser. 
sample thickness. Once we know the integral reflection coefficient, the decrease in the regular constituent, the particle size distribution and the sample thickness, we can evaluate the maximum permissible value of the refractive index on the basis of Eqs. (12), (13), (19), (20) and (25).

The particle size distribution has been obtained through the measurements of remains weight with the sieves. The corresponding results are shown in Fig. $3 \mathrm{a}$.
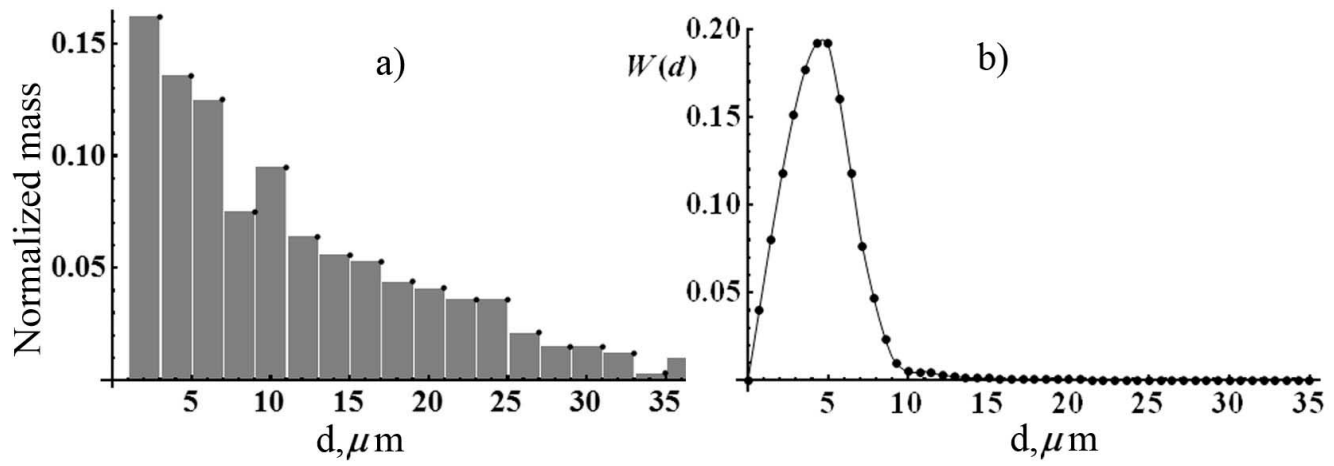

Fig. 3. Weight distribution of the cement particles (a) and qualified quantitative size distribution of the cement particles (b).

The approximate formula for estimating relative amount of particles with the diameter $d_{i}$ and the mass content $w_{i}$ could be derived from the following considerations. Assume that there are $\frac{w_{i}}{\rho_{c e m} \pi d_{i}^{3} / 3}$ particles with the diameter $d_{i}$ in the mass unit of cement, where $\rho_{\text {cem }}$ is the cement density referred to its completely solid stage. According to $[1,2]$, we have $\rho_{c e m}=3120 \mathrm{~kg} / \mathrm{m}^{3}$. So, the relative part under interest is equal to

$$
n_{i}=\frac{w_{i} a_{i}^{-3}}{\sum_{i} w_{i} a_{i}^{-3}}
$$

We have made qualification of the content of particles with the sizes less than $3 \mu \mathrm{m}$ using a microscope. The qualified distribution for the cement powder under analysis is shown in Fig. 3b. Note that the cement brand M-500 has been used in our investigation.

The measurements with the scheme described in Fig. 2 for the case of sample with the thickness of $40 \mu \mathrm{m}$ gives us the transmission coefficient $2.5 \%$ and the integral diffuse reflection and scattering coefficients $66 \%$. Then the values $\rho_{c} \sigma_{t 0} \approx 0.092 \mu \mathrm{m}^{-1}$ $\rho_{c} \sigma_{a} \leq 0.01 \mu \mathrm{m}^{-1}$ might be obtained from Eqs. (10) and (25), respectively. The appropriate sections have been found subject to the particle size distribution given by Eqs. (13). If the real part of the refractive index is considered to be equal to 1.5, then the 
complex refractive index should satisfy the calculated ratio $\frac{\sigma_{t 0}}{\sigma_{a}} \geq 9.2$ and so we get $m \approx 1.5\left(1+1.6 \times 10^{-3} i\right)$.

In order that the diffuse approximation be valid, the condition $\sigma_{s 0} \geq \sigma_{a}$ must be satisfied. The ratio of the sections $\sigma_{s 0}, \sigma_{a}, \sigma_{t 0}$ and the geometrical section of particles is shown in Fig. 4 for different particle sizes and the case of scattered radiation with the wavelength of $0.63 \mu \mathrm{m}$ characteristic for $\mathrm{He}-\mathrm{Ne}$ laser. The values of the refractive index correspond to the cement particles based in air $\left(m \approx 1.5\left(1+1.6 \times 10^{-3} i\right)\right.$, see Fig. $\left.4 \mathrm{a}\right)$ and in water $\left(m \approx 1.13\left(1+1.6 \times 10^{-3} i\right)\right.$, see Fig. 4 b). After analysing the plots in Fig. 4, one can see that the scattering section is larger than the absorption section. This allows us to employ the diffuse approximation method when describing the scattering in concrete.
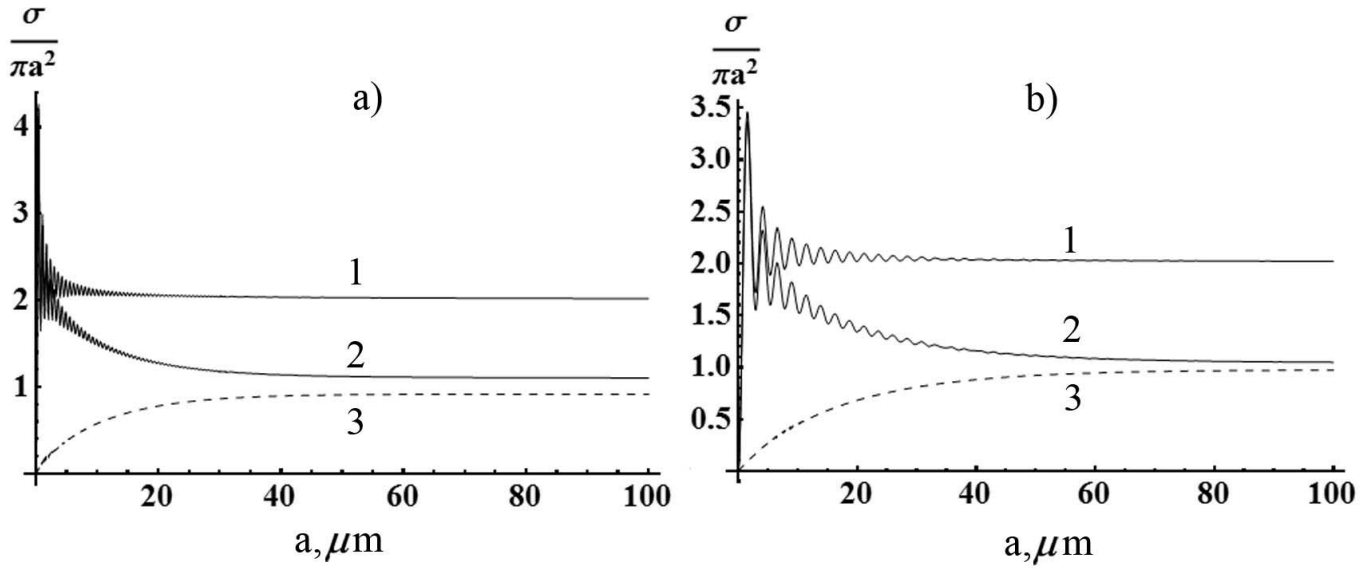

Fig. 4. Dependences of ratios of the extinction section (1), the scattering section (2) and the absorption section (3) to the particle section on the particle radius $(\mu \mathrm{m})$ for the wavelength of $0.63 \mu \mathrm{m}$ and the relative refractive indices $1.5(1+0.0016 i)(a)$ and $1.13(1+0.0016 i)(b)$.

\section{Experiments}

To evaluate diffuse reflection coefficient for the cement solutions it is necessary to find the average cosine of the scattering angle $\bar{\mu}$. The exact calculation of $\bar{\mu}$ for the case of cement with real breakup and the particle shapes differing from spherical one, is very complicated. However, we can evaluate the anisotropy rate for the light scattering by concrete, so that the theoretical calculations on the basis of Eq. (14), with taking Eqs. (13) and (15)-(24) into account, have given us the values of diffuse reflection coefficient $R_{d}$ close to those observed in experiments.

Experimental investigation of backward light scattering of concrete has been performed according to the scheme depicted in Fig. 5. Here a sample 5 is placed inside a chamber 2 with an optical window 6 , through which the sample is illuminated by a laser 4 
(the wavelength of $0.63 \mu \mathrm{m}$ ). The intensity of diffusely scattered radiation is measured with an integral sphere 1. Signals from a light detector 3 come to computer through an analog-to-digital converter. The light beam reflected from the window 6 is disposed outside the integral sphere 1.

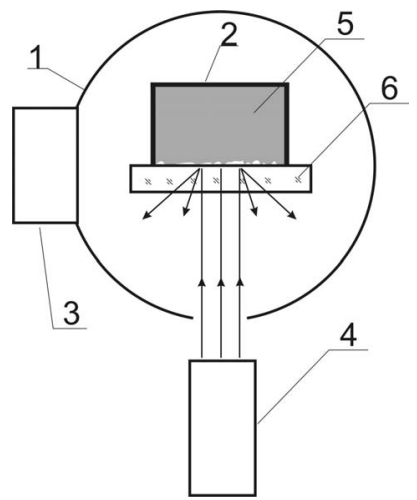

Fig. 5. Scheme for measurements of integral coefficient of the backward scattering for the concrete under hydrogenation:

1 - integral sphere,

2 - chamber,

3 - light detector,

$4-\mathrm{He}-\mathrm{Ne}$ laser,

5 - sample and

6 - optical window.

Table 1. Experimental data for the integral backward light scattering in cement and concrete $(*$ means consideration of the corrected surface reflection)

\begin{tabular}{|l|c|c|c|c|}
\hline & Cement powder & Solid cement & Solid cement in water & $\begin{array}{c}\text { Cement powder } \\
\text { mixed with water }\end{array}$ \\
\hline$R_{d}$ & $24-26 \% *$ & $17-22 \% *$ & $6.5-7.5 \% *$ & $9.5-13 \% *$ \\
\hline
\end{tabular}

Let us first examine the light scattering in the dry cement powder (see Table 1). The diffuse light reflection coefficient for the cement powder is $R_{d}=24-26 \%$. Considering that the powder porosity is equal to $p=0.48[1,2]$, we obtain $\bar{\mu}=-0.0152 \div 0.0765$. So, the scattering in the dry cement is close to isotropic. It is easily seen that, even for the same consignment of cement, the coefficients of backward light scattering differ for different experiments. Form the viewpoint of general considerations, the coefficient $R_{d}$ depends upon size, chemistry and arrangement of particles. Deviation in the $R_{d}$ parameter may be a result of changing average cosine value in the limits mentioned above.

Let us analyse the light scattering in the cement powder with the composition determined above directly after it is mixed with water. One needs to find the parameter $p$. The entire system mass is $1+w$, if the cement mass equals to 1 and $w$ is the water-to-cement ratio (i.e., the ratio of the water and cement masses). Then the water volume is $w \rho_{w}^{-1}$ and the cement volume $\rho_{c e m}^{-1}$. In other words, the parameter $p$ could be calculated with the aid of formula

$$
p=\frac{w \rho_{w}^{-1}}{\rho_{c e m}^{-1}+w \rho_{w}^{-1}}=\frac{w}{\rho_{w} \rho_{c e m}^{-1}+w} .
$$


In particular, we have $p=0.483$ when $w=0.3, \rho_{w}=1000 \mathrm{~kg} / \mathrm{m}^{3}$ and $p=0.46$ when $w=0.275$. However, the porosity of freshly solved cement could not be less than that for the dry powder. That is why, for the case of $w=0.275$ we have also taken the value $p=0.48$, while for the case of $w=0.35$ one has $p=0.52$. Then, under the same assumptions as those made for the dry powder, basing on the diffuse reflection coefficient 9.5-13\% (see Table 1) and the refractive index 1.33 of water, we could get the complex relative refractive index $m=1.13\left(1+1.6 \cdot 10^{-3} i\right)$. Hence, we have $\bar{\mu}=0.592 \div 0.659$, i.e. the cement particles suspended in water scatter light mainly forward. Notice that the deviation of the average cosine is almost the same as that typical for the dry powder $(\Delta \mu \approx 0.07$ ).

Let us now examine the light scattering by completely hydrated cement. Completely hydrated dry cement materials seem to be described by the same complex refractive index, as for the cement powder $\left(m=1.5\left(1+1.6 \times 10^{-3} i\right)\right)$. After hydration of cement materials the porosity decreases, which could be associated with some growth in the particle sizes. In this case we could arrive at the following rule for the crystal radius growth depending on the porosity:

$$
a(p)=a_{0} \sqrt[3]{\frac{(1-p)}{\left(1-p_{0}\right)}},
$$

where $p_{0}$ means the porosity after suspending in water and $a_{0}$ the initial radius of particle.
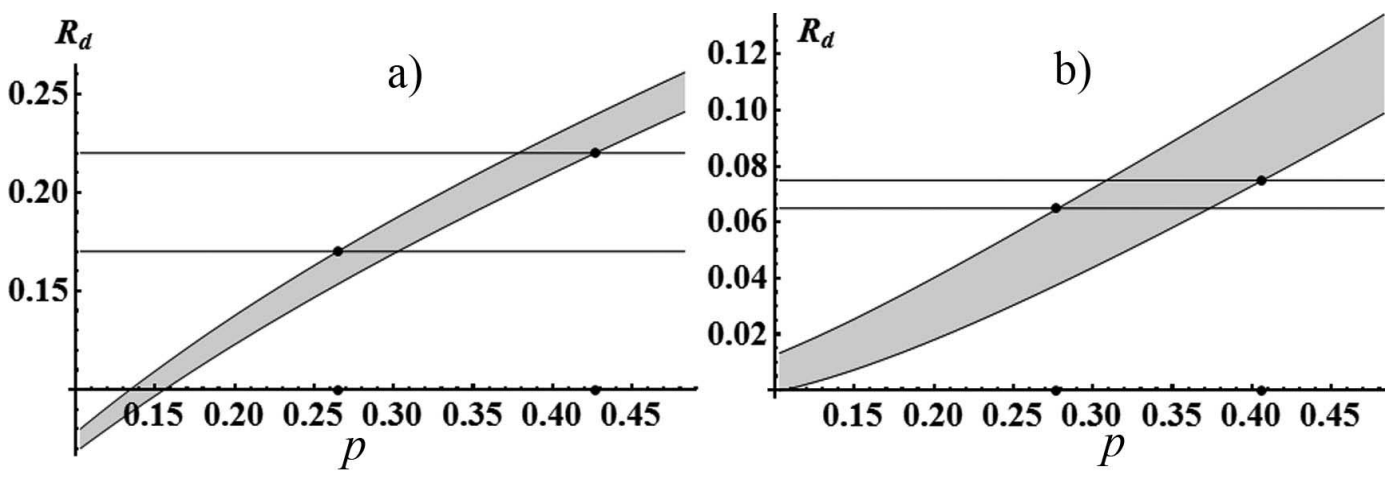

Fig. 6. Dependences of reflection coefficient vs. porosity for the dry and moist concretes in the calculated limits of $\bar{\mu}$ values.

Using particle sizes dependences vs. porosity given by Eq. (28) and Eqs. (13)-(24) we have obtained dependences of the diffuse reflection coefficient vs. the porosity for the dry (Fig. 6a) and moist (Fig. 6b) concretes. They refer to the distribution shown in Fig. 2b and the limits for the $\bar{\mu}$ parameter calculated above. The values measured for the solid concrete (see Table 1) are located between the horizontal lines. It is also seen that the porosity values coincide, thus confirming the right choice of the working method. 
Let us next model the time dependences of diffuse light reflection index during concrete hydration. According to the simplified rule for crystal growth [1], the porosity changes depend on the concrete hydration time $t$ as follows:

$$
p(t)=p_{i} \exp \left(-\left(\frac{t}{\tau}\right)^{\alpha}\right)+p_{f}\left(1-\exp \left(-\left(\frac{t}{\tau}\right)^{\alpha}\right)\right),
$$

While the particles grow, water bounding is happening and, accordingly, the relative refractive index changes, too. Assume that the bounding is ruled the same way as that given by Eq. (29). Then we have the relation between the relative refractive index $m$ and the corresponding average cosine $\bar{\mu}$ of scattering:

$$
\begin{aligned}
& m(t)=m_{i} \exp \left(-\left(\frac{t}{\tau}\right)^{\alpha}\right)+m_{f}\left(1-\exp \left(-\left(\frac{t}{\tau}\right)^{\alpha}\right)\right), \\
& \bar{\mu}(t)=\bar{\mu}_{i} \exp \left(-\left(\frac{t}{\tau}\right)^{\alpha}\right)+\bar{\mu}_{f}\left(1-\exp \left(-\left(\frac{t}{\tau}\right)^{\alpha}\right)\right) .
\end{aligned}
$$

Here $p_{i}, p_{f}, \bar{\mu}_{i}, \bar{\mu}_{f}$ and $m_{i}, m_{f}$ are the initial and final values of the porosity, the anisotropic index of light scattering and the relative refractive index, respectively, $\tau$ denotes the time constant of crystal growth process, and $\alpha$ is the parameter describing time dependence of the relative crystal growth velocity. While modelling the time dependences of diffuse light reflection coefficient, it is necessary to insert the parameters $\bar{\mu}(t)$ and $p(t)$ into Eqs. (13), (14) and (19)-(20), considering Eqs. (15)-(17) and (28). Those dependences refer to the situation when the crystals are generated from the fat solution and they consider no dilution of particles at the initial stage. In any real situation with the formation of cement stone, not all of the water bounds at once. As a consequence, the cement stone includes not only pores filled with air but also those filled with water, crystalline hydrate solutions etc., and even transparent capillaries [1-4].

According to the study [6], measurements of hardening time using the Vicat needle hold on cement solutions with the normal density. Historically (see [1]), the normal density of cement solutions has been obtained experimentally as an optimal integration of good plasticity of the solution with the best mechanical properties of solid concrete. The water-to-cement ratio for getting normal-density cement solution measured according to [6] amounts to $w=0.3$.

During our experiment, we have made concrete samples with different water-tocement ratios $(w=0.275,0.3$ and 0.35 ). Each sample divides in two parts. For one part, the integral diffuse reflection coefficient has been measured and for the other the immersion depth of the Vicat needle has been checked, according to the technique [6]. The dependence of $R_{d}$ upon time is shown in Fig. 7, while the immersion depth of the Vicat needle vs. the time is given in Fig. 8. Here the curves 1, 2 and 3 correspond to $w=0.275, w=0.3$ and $w=0.35$. 

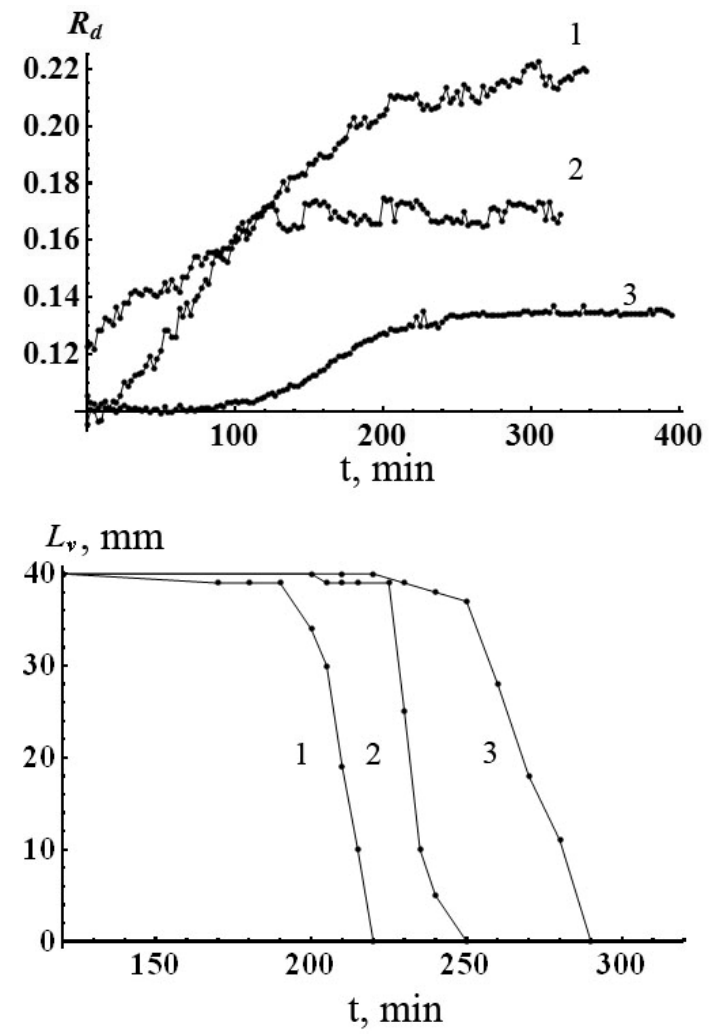

Fig. 7. Experimental dependences of integral diffuse reflection coefficient on the time of concrete hydration for different water-to-cement ratios:

$w=0.275$ - curve 1 ,

$w=0.3$ - curve 2 ,

$w=0.35$ - curve 3 .

Fig. 8. Dependence of immersion depth of the Vicat needle on the time of concrete hydration for different water-to-cement ratios:

$$
\begin{aligned}
& w=0.275-\text { curve } 1, \\
& w=0.3-\text { curve } 2, \\
& w=0.35-\text { curve } 3 .
\end{aligned}
$$

After measuring $R_{d}$ during hydration, final porosity of hydrated samples has been evaluated according to Fig. 6a. For this aim, we have preliminarily dried the samples, since they could contain water remains after hydration. Then we measured the $R_{d}$ value with the scheme of Fig. 5. After drying the samples with $w=0.3$ and 0.275 , the $R_{d}$ value does not differ from those obtained in the end of hydration process (see Fig. 7). At the same time, $R_{d}$ for the dry sample characterised with $w=0.35$ is $17 \%$, while the corresponding value is $13 \%$ in the end of hydration process. That indicates a clear presence of water remains. The average cosine for the dry cement should be $\bar{\mu}=0$, i.e. scattering at the dry cement is isotropic. The refractive index and the average cosine for the samples with $w=0.3$ and 0.275 change in the limits peculiar for the dry and moist cements quoted above. For the sample with $w=0.35$, subject to presence of water remains and evaluation of final porosity, the final average cosine and refractive index have been calculated $\left(\bar{\mu}=0.29\right.$ and $m=1.34\left(1+1.6 \times 10^{-3} i\right)$ ). The final form of theoretical dependence $R_{d}$ on time is determined by the time constant $\tau$ for crystal growth process and the $\alpha$ parameter that describes temporal dependence of the relative crystal growth velocity.

The corresponding experimental and theoretical dependences are shown in Fig. 9 with dots and lines, respectively. Here vertical lines show the beginning and the end of 

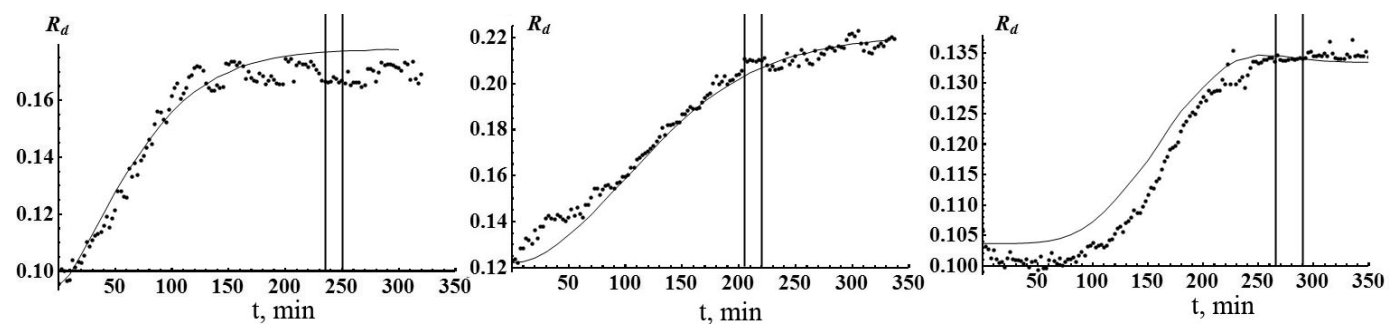

Fig. 9. Experimental (dots) and theoretical (lines) dependences of integral diffuse reflection coefficients on the time for hydrating concrete: $w=0.3$ (a), $w=0.275$ (b), $w=0.35$ (c). Vertical lines show the beginning and the end of hardening derived using the Vicat needle.

hardening detected using the Vicat apparatus. For the cases of $w=0.3, w=0.275$ and $w=0.35$ (see Fig. 9a to Fig. 9c), the constants of crystal growth are respectively $\tau=140 \mathrm{~min}$ and $\alpha=1.5, \tau=180 \mathrm{~min}$ and $\alpha=2$, and $\tau=220 \mathrm{~min}$ and $\alpha=4$.

The results of our modelling correlate fairly well with the experimental data, thus evidencing again the right choice of the method. Small mismatches of experimental and theoretical plots could be explained by a number of simplifications adopted within the model. For instance, dissolving of particles at the initial hydration stage is not taken into account, leading to 'disappearance' of small particles and the corresponding changes in the particle size distribution [1-4]. This effect is shown in Fig. 9c, where reflective index decreases in the beginning. Besides, it is possible that the crystal growth continues after bounding of all water [1-4]. Then the constants $\tau$ and $\alpha$ governing the growth process (see Eq. (29)) and the water bound processes (see Eqs. (30) and (31)) are different. This effect is shown in Fig. 9b and Fig. 9a. Taking these effects into account would have complicated our model calculations drastically and, in particular, would have needed introducing additional parameters.

In 45 days after induration the samples under analysis have been tested on its compression strength. For the sample with The destructive pressures are $46 \mathrm{MPa}, 33 \mathrm{MPa}$ and $38 \mathrm{MPa}$ for $w=0.3, w=0.275$ and $w=0.35$, respectively. This means that the concrete made of cement grout with the normal density is the most pressure-proof.

\section{Conclusions}

The measurements based upon the Vicat apparatus have shown that the induration time is in a direct dependence on the amount of water. Furthermore, the induration time decreases if this amount does, and vice versa. However, the method does not provide information about the crystal growth that binds concrete. The optical method consisting in measurements of the light backscattering coefficient enables one to study the processes of crystal growth and bonding of water with hydratable minerals.

The analysis of modelling results testifies that the normal cement grout density ( $w=0.3$ ) is associated with the smallest time constant of crystal growth and, accordingly, the smallest water bonding velocity (see Fig. 9a). From the point of view of chemistry, 
this implies that the optimal conditions for crystal growth are just reached. As seen from Fig. 9, the crystal growth has finished long before the induration start detected with the Vicat apparatus. So, the induration in this case progresses without mechanical stresses, thus influencing positively final mechanical properties of the concrete. At the same time, slowing-down of the crystal growth occurs when the water amount is less ( $w=0.275$, see Fig. 9b). The crystals continue to grow even after finish of the induration detected with the Vicat apparatus. This leads to arising additional mechanical stresses, which worsen mechanical properties of the concrete. Finally, the highest amount of water $(w=0.35)$ also slows the crystal growth and a part of water remains unbounded. Crystal growth is then finished almost simultaneously with the beginning of concrete setting, according to the Vicat technique. However, it follows from the results obtained with the Vicat technique that the induration process itself takes more time. In other words, the excess of water imposes formation of less durable crystals, thus worsening mechanical properties of the concrete.

The optical technique presented above allows performing earlier diagnostics of concrete hardening process, when compare with the Vicat needle technique and, moreover, it carries additional information about the crystal growth process. Nevertheless, it is very difficult to evaluate the time of mechanical induration of concrete, unlike the Vicat apparatus. Therefore the most complete information about the concrete hydration may be provided when combining our optical diagnostics with the Vicat measurements.

\section{References}

1. Gorskiy VF, Plugging materials and solutions. Handbook. Chernivtsi: Oblpoligrafvydav (2006).

2. Lee F M, The chemistry of cement and concrete, 3rd ed., New York: Chemical Publishing Company (1971).

3. Ramachandran V S and Beaudoin J J, Handbook of analytical techniques in concrete, National Research Council of Canada, New York: William Aandrew Publishing, LLC (2001).

4. Bulatov A I and Danyushevskiy V S, Plugging materials reference manual. Moscow: Nadra (1987).

5. Ferraris CF, Hackley V and Aviles A I, 2004. Measurement of particle size distribution in Portland cement powder: analysis of ASTM round robin studies. Cement, Concrete and Aggregates. 26: 1-11.

6. GOST 310.3-76. Cements. Methods of definition of normal density, terms of harden and uniformity of change of volume.

7. GOST 310.1-76. Cements. Test methods. General provisions.

8. Gorsky M P, Gorskiy V F, Maksimyak P P and Gorskiy P V, 2006. Study of cement hydration by coherent light scattering. Equip.Techn. for Oil and Gas Ind. 5: 42-45. .

9. Gorsky M P, Maksimyak A P and Maksimyak P P, 2007. Study of speckle-field 
dynamics scattered by surface of concrete during congelation. Proc. SPIE 6635: 66350E.

10. Ishimaru A, Wave propagation and scattering in random media. Vol.1, 2. New York: Academic Press (1978).

11. Born M and Wolf E, Principles of optics. New York: Cambridge: University Press (1999).

12. Akkermans E, Wolf P E, Maynard R and Maret G, 1988. Theoretical study of the coherent backscattering of light by disordered media. J. Phys. France 49: 77-98.

13. Jos Stam, Multiple scattering as a diffusion process. Eurographics Rendering Workshop (1995).

14. Morse P M and Feshbach H, Methods of theoretical physics. New-York: McGrawHill (1953).

Gorsky M.P., Maksimyak P.P. and Maksimyak A.P. 2009. Studies of light backscattering at concrete during its hydration. Ukr.J.Phys.Opt. 10: 134-149.

Анотація. У даній роботі нами теоретично і експериментально досліджено зміни сумарного коефіџієнта відбивання бетону під час його гідратації. Для опису розсіяння світла бетоном, у прочесі його гідратаиії, був використаний метод дифузного наближення, а результати були порівняні з отриманими експериментальними даними. Запропонований нами оптичний метод дозволяє забезпечувати діагностику ранніх стадій гідратаиії при затвердінні бетону і передбачити його механічні властивості. 